\title{
An Improved Formation Control Algorithm in Vicon 3D Motion Capture System
}

\author{
Qiu Tang, Chenxiao Cai ${ }^{*}$ and Lei Ma \\ Nanjing University of Science and Technology, Nanjing, 210094, China \\ ${ }^{*}$ Corresponding author
}

\begin{abstract}
In this paper, an improved leader-follower configuration control algorithm is proposed to solve the formation problem in Vicon 3D motion capture system. Firstly, an appropriate velocity filter with weight is designed and the acceleration gain matrix is reconstructed to reduce noise and oscillations caused by velocity and acceleration computed by discrete position. Secondly, using Sliding Model Control (SMC) for formation, damping parameters are added to the signal of tracking control for each single quadrotor. Thirdly, formation flight with two quadrotors is carried out. Finally, the experiment results show the feasible and effectiveness.
\end{abstract}

Keywords-quadrotor; PD algorithm; circular formation; sliding mode control; leader-follower

\section{INTRODUCTION}

Recently, quadrotor with the capability of vertical take-off and landing is one of the hot research spots. Multiple quadrotors cooperative formation flight has obvious advantages in success rate improving, efficiency increasing and cost reducing of tasks such as rescue search, military reconnaissance. Many effective methods have been proposed to solve the control problem, which are mainly based on the leader-follower strategy [1-4], virtual structure method [7], and behavior-based approach [8].

The advantages of virtual structure method are simplicity and robustness [15]. However, it is disadvantageous for that any single failure will lead to failure of the whole mission. In behavior-based approach, decentralization is its main advantage [16], but the approach lacks for mathematical modeling. Therefore, the simplicity and reliability properties contribute to the popularity of the leader-follower strategy.

Classical application of cooperation formation flight under the leader-follower strategy is to finish the search and rescue missions. The leader is used to transport much-needed drugs [3], as well as to provide feasible route map for the followers by detecting the surrounding environment. Meanwhile, the followers' tasks are to carry other necessities and follow the leader.

In the formation flight with leader-follower strategy, many feasible methods, such as back-stepping technique [4] and sliding model control (SMC) [1-3], have been studied in many ways. Back-stepping technique can effectively reduce the calculation time of formation control, but it is not an accurate controller. SMC has shown its robustness and accuracy in the use of formation flight, thus, it was used in many research works. In [1], SMC algorithm is used to complete the formation flight. Despite the obvious advantages, the leader is "virtual leader", which ignores the influence of actual factors. The leader's actual flight path is not so perfect that the actual formation control performance is reduced. However, SMC method has two disadvantages: high computation time as well as other non-linear controller methods, and the chatting effect. In [2], linear quadratic regulator (LQR) is used in the position control of quadrotor to reduce the chatter caused by SMC algorithm, and the results are verified by the simulation, but not been carried out by the experiment. In [3], the switching function with smooth weight is used to smooth the control signal created by SMC, and the experiment is finished with two quadrotors. But the quadrotors tracking control with PD algorithm ignores the discrete position provided by Vicon motion capture system. The velocity computed by discrete position using the simple derivative caused noise and chatters [4]. In the condition of the derivative of a non-zero velocity gain matrix, the phenomenon of the speed fluctuation is obvious, especially in hover control [5]. In [5], the author focused on the formation flight experiment platform development and trajectory tracking, but formation control was not presented.

In this paper, an improved algorithm for formation control with leader-follower strategy is proposed. PD algorithm is used for tracking control, and a filter of velocity is designed to reduce the chatter, especially for the follower. SMC is used for formation control, while damping parameters are added such to smooth the quadrotors trajectory tracking.

\section{HARDWARE AND SOFTWARE IMPLEMENTATION}

The development and experiment of formation cooperative flight are based on indoor navigation device, that is, Vicon 3D motion capture system. The whole system includes the Vicon system, Pixhawk quadrotors, ground-station- server-PC equipped with MATLAB, Visual C++ 2012, the wireless communication device XBEE, RF joysticks used for emergencies, as shown in Fgure 1. Vicon motion capture system allows the user to acquire translation and rotation data of quadrotors through the reflective marks, and provides a convenient software interface, the acquisition rate of $200 \mathrm{~Hz}$ meeting the real-time requiremenets and positioning accuracy within $1 \mathrm{~mm}$. Users apply Tracker to establish quadrotors rigid models, as shown in Figure II. XBEE is used to carry out the wireless transmission of information, regarding the Vicon motion capture system as position control feedback of the 
quadrotors. It was proved that the DIY quadrotors can be used for formation flying [5], users can change the control program of Pixhawk quadrotors according to the experimental requirements [10]. At the same time, Pixhawk flight controller is equipped with a variety of flight modes, which can effectively reduce the risk of the experiment.

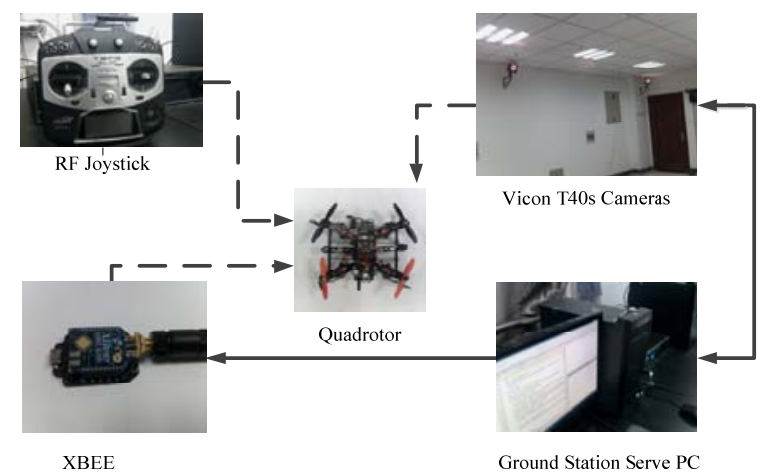

FIGURE I. FULL SYSTEM.

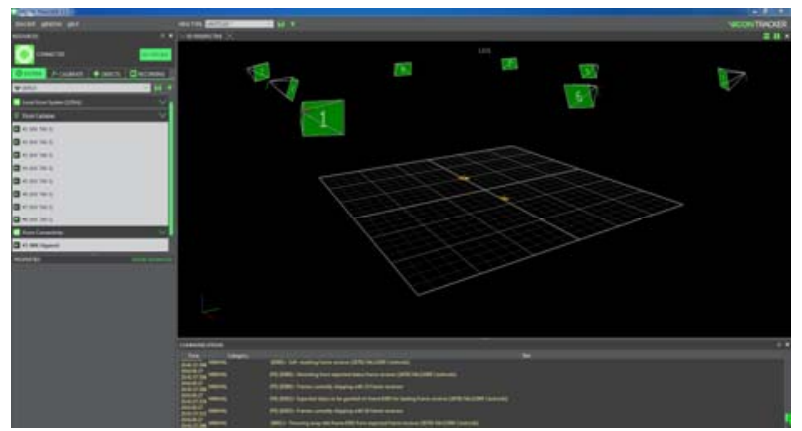

FIGURE II. TRACKER INTERFACE.

Due to environmental constraints of the system, the indoor flight area is limited to $5 \mathrm{~m} \times 5 \mathrm{~m} \times 2.7 \mathrm{~m}$. In order to reduce the influence of the shock of marks for quadrotors as much as possible, a relatively stable attaching method is adopted.

\section{KINEMATIC MODEL OF QUADROTOR}

The quadrotor can be seen as a rigid body in space, and the motion of the rigid body is driven by gravity and aerodynamic force (see Figure III). In Figure I, $E$ represents inertial coordinate while $B$ is a body fixed coordinate frame.

In [6], the quadrotor kinematics model is obtained by Newton-Euler equations as following:

$$
\left[\begin{array}{cc}
m J_{3 \times 3} & 0 \\
0 & J
\end{array}\right]\left[\begin{array}{c}
\dot{V} \\
\dot{\omega}
\end{array}\right]+\left[\begin{array}{c}
\omega \times m V \\
\omega \times J \omega
\end{array}\right]=\left[\begin{array}{c}
F \\
\tau
\end{array}\right]
$$

where $m$ is the quadrotor mass, $J$ is a quadrotor inertias diagonal matrix. $V$ and $w$ are the linear velocity and angular velocity in quadrotor body coordinates, respectively. $F$ and $t$ are the thrust and torque applied to the quadrotor by motors ,respectively.

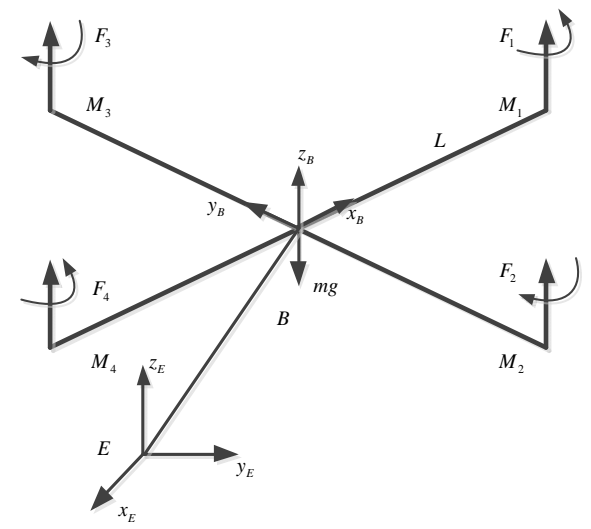

FIGURE III. QUADROTOR CONFIGURATION WITH INERTIAL AND BODY-FIXED FRAME

The position $x, y, z$ and direction $j, q, y$ of the quadrotor are determined by the inertial coordinate $E$ (see Figure I). The transformation from the inertial coordinate frame $E$ to the body coordinate frame $B$ is obtained by Euler rotation as following:

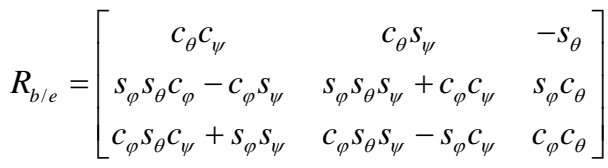

$c_{i}$ and $s_{i}$ refer to $\cos (i)$ and $\sin (i)$, respectively, where $i$ can be Euler angles $j, q$ or $y$ which represent roll, pitch and yaw, respectively. The attitude vector of the quadrotor is defined in the body coordinate system as:

$$
\Omega=\left[\begin{array}{l}
p \\
q \\
r
\end{array}\right]
$$

where $p, q$ and $r$ represent roll, pitch and yaw angles in the quadrotor body frame, respectively. According to the principle of coordinate transformation, the kinematic relation of generalized velocities and the angular velocities can be shown as:

$$
\left[\begin{array}{c}
\dot{p} \\
\dot{q} \\
\dot{r}
\end{array}\right]=\left[\begin{array}{ccc}
1 & 0 & -s_{\theta} \\
0 & c_{\psi} & s_{\varphi} c_{\theta} \\
0 & -s_{\varphi} & c_{\varphi} c_{\theta}
\end{array}\right]\left[\begin{array}{c}
\dot{\varphi} \\
\dot{\theta} \\
\dot{\psi}
\end{array}\right]
$$

Define input variables as $U_{1}-U_{4}$ as following: 


$$
\left[\begin{array}{c}
U_{1} \\
U_{2} \\
U_{3} \\
U_{4}
\end{array}\right]=\left[\begin{array}{c}
F_{1}+F_{2}+F_{3}+F_{4} \\
\left(F_{3}-F_{4}\right) L \\
\left(F_{2}-F_{1}\right) L \\
M_{3}-M_{1}+M_{4}-M_{2}
\end{array}\right]
$$

where $U_{1}$ is the total output of the four motors with propeller blades, $U_{2}$ is roll torque, $U_{3}$ is pitch torque, $U_{4}$ is yaw torque, $L$ is distance from the center of the motor to the geometric center of quadrotor, $F_{i}, i=1, \ldots, 4$ is the thrust generated by the $i$ th propeller, ${ }^{M_{j}}, j=1, \ldots 4$ is the torque generated by the $j$ th propeller.

Thus, a simplified quadrotor's kinematic model is obtained from the equation (1)-(5):

$$
\left[\begin{array}{c}
\ddot{x} \\
\ddot{y} \\
\ddot{z} \\
\ddot{\varphi} \\
\ddot{\theta} \\
\ddot{\psi}
\end{array}\right]=\left[\begin{array}{c}
\frac{\left(c_{\psi} s_{\theta} c_{\varphi}+s_{\psi} s_{\varphi}\right) U_{1}}{m} \\
\frac{\left(s_{\psi} s_{\theta} c_{\varphi}-c_{\psi} s_{\varphi}\right) U_{1}}{m} \\
\frac{\left(c_{\theta} c_{\varphi}\right) U_{1}-m g}{m} \\
\frac{U_{2}}{J_{x x}} \\
\frac{U_{3}}{J_{y y}} \\
\frac{U_{4}}{J_{z z}}
\end{array}\right]
$$

where $g$ is local gravity acceleration.

\section{Control System Design}

In this section, control system design can be divided into two sections: (i) the trajectory tracking control (ii) circular formation control.

\section{A. Trajectory Tracking Control}

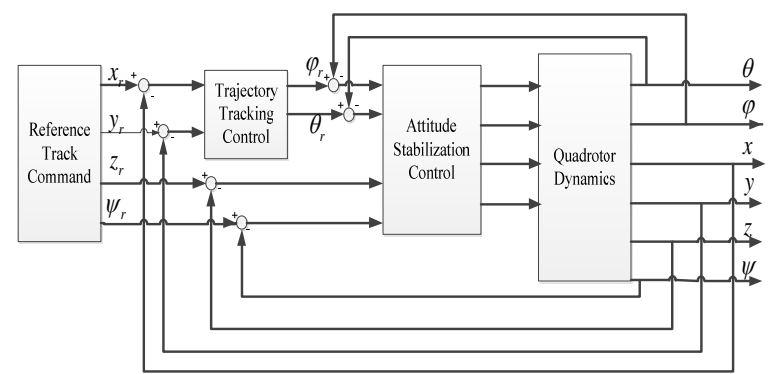

FIGURE IV. QUADROTOR CONTROL SYSTEM
Figure IV shows a traditional control system structure of the quadrotor, which includes position control and attitude control, as described above. Current quadrotor attitude control has been quite mature, since many research efforts have been spent on both theory developments and practice experiments [12-14]. In this section, we focus on the position control of quadrotors.

The linearized model of the position control is:

$$
\ddot{x}=\theta g
$$

$$
\ddot{y}=-\varphi g
$$

In order to complete the non-smooth trajectory tracking of the quadrotor in formation flight, the damping parameters are added to the expected output. PID controller is used to calculate $a$ and as following:

$$
\begin{gathered}
e_{p}=\xi-\xi_{r} \\
e_{v}=\dot{\xi}-\dot{\xi}_{r} \\
a=-K_{p} e_{p}-K_{i} \int e_{p} d t-K_{d} e_{v}-K_{d p} \dot{e}_{p}-K_{d v} \dot{e}_{v}+\ddot{\xi}_{r}
\end{gathered}
$$

where $e_{p}$ and $e_{v}$ are the position error and velocity error, respectively, $K_{p}, K_{i}$ and $K_{d}$ are corresponding gain matrixes, $K_{d p}$ and $K_{d v}$ are gain matrixes of damping parameters, ${ }^{x}$ and $x_{r}$ are the current and reference position, ${ }^{\&}$ and ${ }_{r}^{\&}$ are the current and reference velocity, ${ }_{r}$ is the reference acceleration feed forward. In addition, DIY quadrotor is not absolutely symmetrical, and the calibration of the electronic speed control is not exactly the same that there may be a small offset in position control. In view of this, the offset can be eliminated by adding an integral term.

A common problem is that computing velocity through the discrete position by simple derivative will lead to noise and vibration when using PD algorithm. Experiments have shown that this will contribute to chatters or even instability [5], [11. On the one hand, the acceleration gain matrix obtained by derivative of velocity is set to zero. As a result, chatters can be reduced [4], [5]. On the other hand, velocity can be obtained by using a predetermined point closet to the current point, therefore smoothness of the trajectory tracking of the quadrotor is achieved [5]. However, in this algorithm, the follower's future navigation point is unknown. So it can only calculated form the leader. In this condition, the leader's fluctuation will directly lead to the fluctuation of the follower, especially in 
velocity. Taking into account the fluctuation of the velocity is closely related to the front of several velocities. So, reference speed of the followers can be calculated as:

$$
v_{r}^{F}=\lambda_{1} v^{F}(k-2)+\lambda_{2} v^{F}(k-1)+\lambda_{3} v^{F}(k)
$$

where $v_{r}^{F}$ is the reference velocity of the follower after filtering, $v^{F}(k-2)$ and $v^{F}(k-1)$ are the follower velocity of the first two sampling time, respectively, $v^{F}(k)$ is the current reference velocity of the follower, $l_{i}, i=1, \ldots, 3$ are the weights and they satisfy the following relationship:

$$
\left|\lambda_{1}\right|+\left|\lambda_{2}\right|+\left|\lambda_{3}\right|=1
$$

Adjusting the weights for refer velocity filtering can achieve the fluctuation reduction of the followers' trajectory tracking.

\section{B. Circular Formation Control}

The curve trajectory is crucial for the quadrotor to avoid obstacles and flight turning, so circular formation strategy and SMC formation control algorithm proposed in [3] is utilized in this subsection. This strategy is to control the distance between the leader and follow through the center (indoor origin) and also the angle $a_{L F}$ to complete the formation flight, as shown in Figure V.

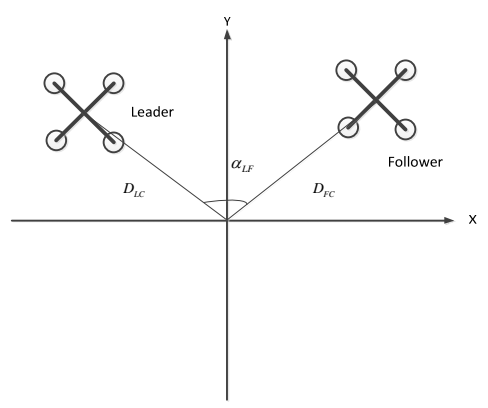

FIGURE V. TWO QUADROTORS FORMATION STRATEGY

The relationship between leader and follower is as following:

$$
\begin{aligned}
& x_{F}=\frac{A}{\sqrt{1+B^{2}}} \\
& y_{F}=x_{F} \times B \\
& A=D_{L F}-\sqrt{x_{L}^{2}+y_{L}^{2}} \\
& B=\tan \left(-\alpha_{L F}+\tan ^{-1}\left(\frac{y_{L}}{x_{L}}\right)\right)
\end{aligned}
$$

where $x_{L}$ and $y_{L}$ are the $x$-axis and $y$-axis position measurements of the leader in inertial coordinate, $x_{F}$ and $y_{F}$ are the $x$-axis and $y$-axis reference position of the follower in the inertial coordinate according to the formation requirement, $D_{L F}$ is the distance through the center between the leader and the follower. The relationship between $D_{L F}$ and $D_{L C}$ with $D_{F C}$ is as following:

$$
D_{L F}=D_{L C}+D_{F C}
$$

Figure VI describes the control structure of formation control in this paper. As mentioned above, this paper uses SMC for formation control proposed in [3].

The SMC of formation flight in this subsection can be calculated in the method proposed in [3] with:

$$
u_{n l}=-K f(\sigma)
$$

$$
f(\sigma)=\frac{\sigma}{|\sigma|+\delta}
$$

where $u_{n l}$ is control input, $K$ is the switching amplitude, $s$ is sliding surface, $f(s)$ is smoothing function to reduce chatting, and $d$ is the smooth weight.

However, conventional PID algorithm is used in tracking control, while discrete characteristics of position in the system are not taken into consideration in [3]. Here, it improves the follower tracking the leader according to the content of the subsection A. Damping parameters ensure the follower can track the leader to maintain formation. Moreover the processing of velocity items improves the ability of attenuating noisy and chattering.

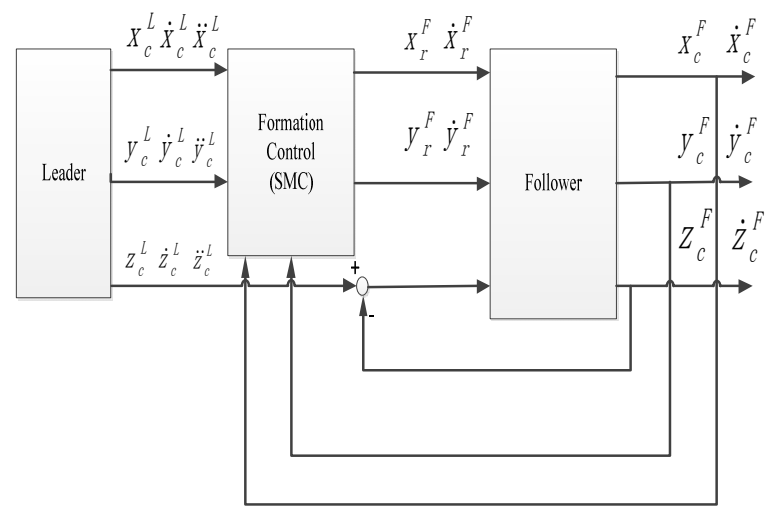

FIGURE VI. FORMATION CONTROL SYSTEM 


\section{EXPERIMENTAL RESULTS}

In order to prove the validity of the proposed control algorithm, the formation flight with circular leader-follower strategy is applied to two quadrotors.

The parameters of Pixhawk DIY quadrotor used are shown in Table I.

TABLE I. PIXHAWK DIY QUADROTOR PARAMETERS

\begin{tabular}{ccc}
\hline \hline Symbols & Values & Units \\
$\mathrm{m}$ & 0.79 & $\mathrm{~kg}$ \\
$\mathrm{~g}$ & 9.794 & $\mathrm{~m} / \mathrm{s}^{2}$ \\
$\mathrm{~L}$ & 150 & $\mathrm{~mm}$ \\
$\mathrm{~J}_{\mathrm{xx}}$ & 0.0031 & $\mathrm{~kg} \cdot \mathrm{m}^{2}$ \\
$\mathrm{~J}_{\mathrm{yy}}$ & 0.0031 & $\mathrm{~kg} \cdot \mathrm{m}^{2}$ \\
$\mathrm{~J}_{\mathrm{zz}}$ & 0.00609 & $\mathrm{~kg} \cdot \mathrm{m}^{2}$ \\
Flying Time & 10 & $\mathrm{~min}$ \\
Max Speed & 12 & $\mathrm{~m} / \mathrm{s}$ \\
\hline \hline
\end{tabular}

$\overline{\text { According to Figure VII, the trajectories of the leader }}$ and the follower achieve a circular formation. The circular formation is centered at $(0,0)$ with a radius of about $1.0 \mathrm{~m}$.

Figures VIII and IX show the $x$-axis and ${ }^{y}$-axis trajectory, respectively, when using simultaneous angle and distance control strategy. The solid line is the leader's trajectory while the dashed line is the follower's trajectory and the dotted line is the follower's reference trajectory generated by (14). From Figures VIII and IX, Figure VIII and Figure IX show the smoothness trajectories in both $x$-axis and ${ }^{y}$-axis, which satisfies our design requirement.

Remark 1 The advantage of Vicon capture system is its high precision positioning of indoor rigid body. In formation flight to formation reconstruction and path planning according to the position of the obstacle will be the next focus of the work in the future. Similarly, the research content of indoor formation flying to the outside also not be ignored.

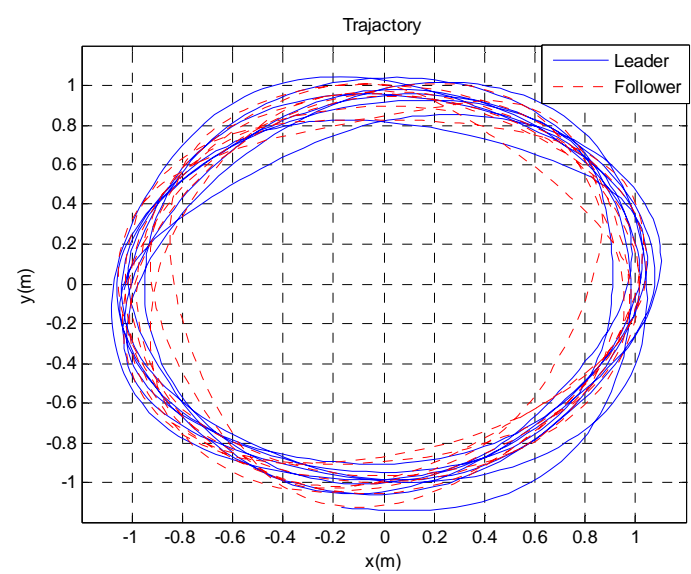

FIGURE VII. FORMATION FLIGHT TRAJECTORY

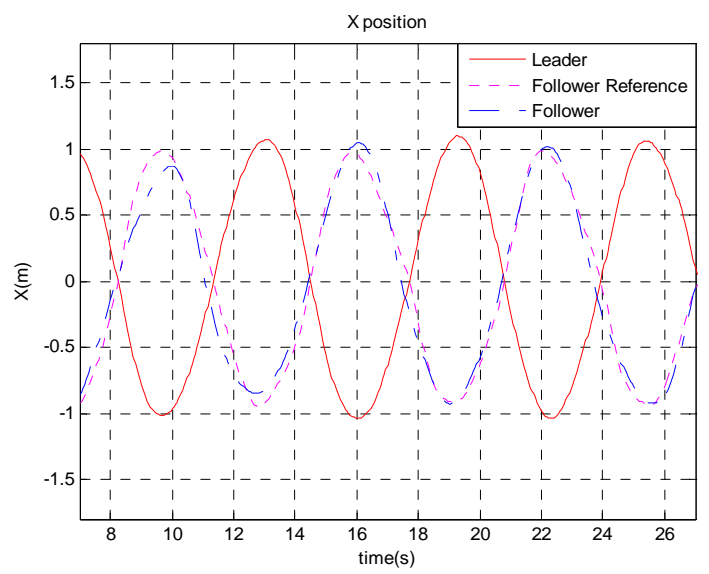

FIGURE VIII. X POSITION

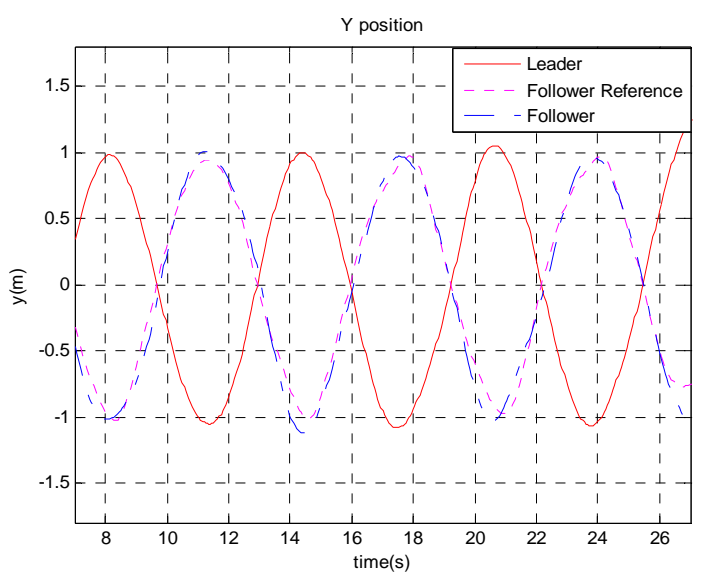

FIGURE IX. Y POSITION

\section{CONCLUSIONS}

In this paper, it is discussed the leader-follower formation control problems for quadrotors. An improved formation control algorithm in Vicon motion capture system was proposed. Chattering problem caused by the discontinuous switching characteristics of SMC was improved by adding damping parameters to the signal of tracking control, while chattering problem caused by PD algorithm in position control was solved by using an appropriate velocity filter with weight and setting the gain matrix of the derivative of velocity to zero. Practical experiment has been carried out by our DIY quadrotors. In addition, the formation flight based on Pixhawk in the Vicon capture system, it is provided a concise and valid implementation method.

\section{REFERENCES}

[1] D. A. Mercado, R. Castro and R. Lozano. "Quadrotors flight formation control using a leader-follower approach.” European Control Conference, Zurich, Switzerland(2013): 3858-3863.

[2] Khaled A. Ghamry, Youmin Zhang. "Formation Control of Multiple Quadrotors Based on Leader-fllower Method.” International Conference on Unmanned Aircraft Systems(2015): 1037-1042.

[3] M.Fadhil Abas, Dwi Pebrianti, Syaril A Zrad, D. Iwakura, Yuze Song, and K. Nonami. "Circular formation control of multiple quadrotor aerial 
vehicles.” Intelligent Systems, Control and Automation,Japan, Springer(2013): 109-132.

[4] YX Min, KC Cao, HH Sheng, Z Tao. "Formation tracking control of multiple quadrotors based on backsrepping.” 34th Chinese Control Conference(2015): 4424-4430

[5] Edwin Davis, Ben E.Nizette, Changbin Yu. "Development of a low cost quadrotor platform for swam experiments." 32th Chinese Control Conference(2013):7072-7077.

[6] Samir Bouabdallah, Pierpalolo Murrieri and Roland Siegwart. "Design and control of an indoor micro quadrotor.” 2004 IEEE International Conference on Robotics \& Automation(2004): 4393-4398.

[7] Dingjiang Zhou and Mac Schwager. "Virtual rigid bodies for coordinated agile maneuvering of teams of micro aeria vehicles.” IEEE International Conference on Robolicts and Automation(2015):17371742 .

[8] Brad S, Gu Y,Marcello R N,et al. "3-Aircraft formation flight experiment." 14th Mediterranean Conference on Control and Automation(2006):1-6.

[9] Harry Berghuis, Henk Nijmeijer. "Global regulation of robots using only position measurements." Systems \& Control Letters 21, NorthHolland(1993): 289-293.

[10] Arducopter.[Online].Available: https://github.com/ArduPilot/ardupilot/tree/master/ArduCopter(2016).

[11] D.Mellinger and V. Kumar. "Minimum snap trajectory generation and control for quadrotors.” IEEE International Conference, Robotics and Automation(2011):2520-2525.

[12] Jing-Jing Xiong and En-Hui Zheng. "Position and attitude tracking control for a quadrotor UAV.” ISA Transactions 53(2014):725-731

[13] Zhogyu Zuo and Pengkai Ru. Augmented. "L1 adaptive tracking control of quadrotor unmanned aircrafts." IEEE Transations on aerospace and electronic systems 50.4(2014):3090-3101.

[14] Guiherme V. Raffo, Manuel G. Ortega and Francisco R. Rubio. "Robust nonlinear control for path tracking of a quadrotor helicoptor." Asian Journal of Control 17.1(2015):142-156.

[15] N. E. Leonard and E. Fiorelli. "Virtual leaders, artificial potentials and coordinated control of groups." 40th IEEE Conference on Decision and Control(2001):142-156.

[16] T.Balch and R. C. Arkin. "Behavior-based formation control for multirobot teams." IEEE Transactions on Robotics and Automation 14.6(1998):142-156. 